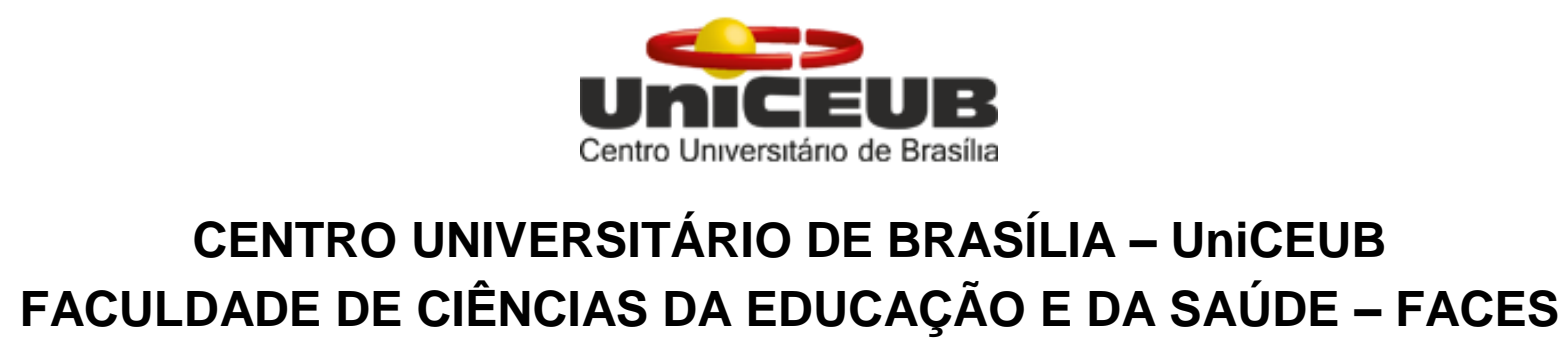

PROGRAMA DE INICIAÇÃO CIENTÍFICA

LUÍZE FOIZER FILGUEIRA

MARINA DA COSTA RIBEIRO

ESTUDO DA PREVALÊNCIA DA RESISTÊNCIA INSULÍNICA E SUA RELAÇÃO COM O PERFIL LIPÍDICO E A SÍNDROME METABÓLICA, EM PACIENTES DO LABORATÓRIO ESCOLA DO UNICEUB NO DISTRITO FEDERAL

BRASÍLIA

2017 


\section{UnTC=UB \\ Centro Unıversitárıo de Brasílıa \\ LUÍZE FOIZER FILGUEIRA \\ MARINA DA COSTA RIBEIRO}

\section{ESTUDO DA PREVALÊNCIA DA RESISTÊNCIA INSULÍNICA E SUA RELAÇÃO COM O PERFIL LIPÍDICO E A SÍNDROME METABÓLICA, EM PACIENTES DO LABORATÓRIO ESCOLA DO UNICEUB NO DISTRITO FEDERAL}

Relatório final de pesquisa de Iniciação Científica apresentado à Assessoria de Pós-Graduação e Pesquisa pela Faculdade de Ciências da Educação e da Saúde - FACES.

Orientação: Msc. Vanessa Carvalho Moreira

BRASÍLIA 


\title{
ESTUDO DA PREVALÊNCIA DA RESISTÊNCIA INSULÍNICA E SUA RELAÇÃO COM O PERFIL LIPÍDICO E A SÍNDROME METABÓLICA, EM PACIENTES DO LABORATÓRIO ESCOLA DO UNICEUB NO DISTRITO FEDERAL
}

\author{
Marina da Costa Ribeiro - UniCEUB, PIC voluntário \\ marinacrib@hotmail.com \\ Luíze Foizer Filgueira - UniCEUB, PIC voluntário \\ lufoizer@gmail.com
}

Vanessa Carvalho Moreira - UniCEUB, professora orientadora vanessa.moreira@uniceub.br

A resistência insulínica $(\mathrm{Rl})$ consiste na dificuldade da insulina em exercer suas ações no metabolismo de gordura e na retirada de glicose do sangue para as células. Geralmente está associada a obesidade, diabetes e problemas cardiovasculares. $\mathrm{O}$ número de casos de RI tem aumentado consideravelmente nos últimos anos, principalmente quando associado à Síndrome Metabólica (SM). O objetivo desta pesquisa é analisar a prevalência dos casos de resistência insulínica e sua relação com o perfil lipídico no desenvolvimento da síndrome metabólica na população do Distrito Federal, entre os anos de 2015 e 2016. Trata-se de um estudo transversal observacional descritivo no qual foram analisados 217 prontuários da base de dados do laboratório-escola de análises clínicas do Centro Universitário de Brasília UniCEUB. Foram considerados dados epidemiológicos (sexo e idade) e os resultados dos exames de glicemia em jejum, hemoglobina glicada, teste oral de tolerância a glicose (TOTG) e perfil lipídico. A prevalência de RI foi de 30,41\%, considerando o TOTG como exame diagnóstico. Quanto ao perifl lipídico o HDL baixo foi o fator mais frequente $(60 \%$ dos casos), seguida de elevação do colesterol total $(57 \%)$ e triglicerídeos (31\%). A resistência insulínica apresentou forte correlação com alteração no perfil lipídico e esteve presente em $27 \%$ dos casos avaliados. A Síndrome metabólica, seguindo os parâmetros da Sociedade Brasileira de Endocrinologia, representou $12 \%$ dos dados analisados. Os resultados obtidos, sugerem a importância da realização de intervenções clínicas visando esclarecer, prevenir e orientar a população para o impacto da RI na saúde e os fatores de risco a ela associados, estimulando um estilo de vida saudável, com prática de atividade física, controle alimentar e redução do peso.

Palavras-Chave: Resistência insulínica. Hipertrigliceridemia. Síndrome metabólica. 


\section{SUMÁRIO}

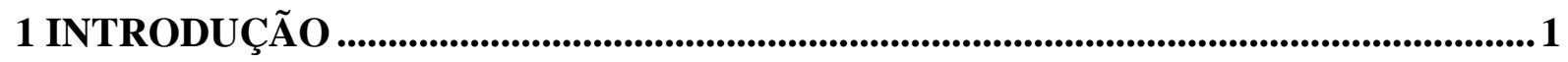

2 OBJETIVOS ............................................................................................................................ 2

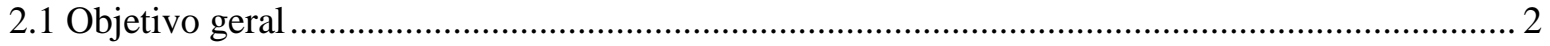

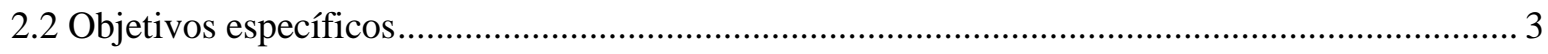

3 FUNDAMENTAÇÃO TEÓRICA ...........................................................................3

4 METODOLOGIA ....................................................................................................................5

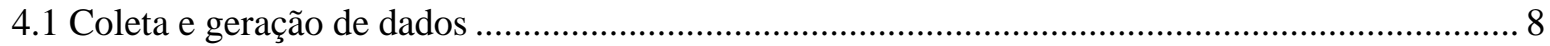

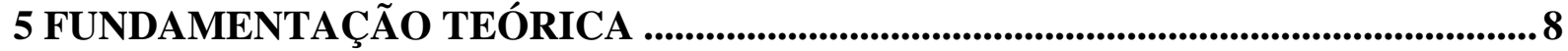

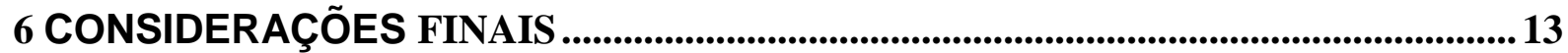

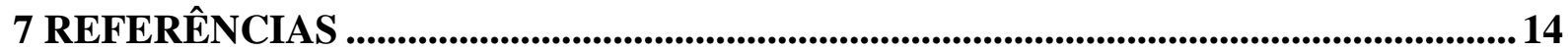




\section{INTRODUÇÃO}

O desenvolvimento tecnológico e a escassez de tempo cada vez mais frequente, vem alterando o modo de vida dos indivíduos, tais fatores contribuem cada dia mais para um estilo de vida sedentário com alto nível de estresse emocional e alimentação desbalanceada (LOPES, 2009). Tais comportamentos acarretam alterações significativas na saúde das pessoas, como a obesidade, a resistência insulínica (RI), alterações no perfil lipídico e a síndrome metabólica (SM). O país vem apresentando um quadro alarmante em relação a diabetes, doenças cardiovasculares e o número de óbitos a elas relacionado, onde a resistência insulínica e a síndrome metabólica podem ter forte correlação (CAMPOS et al, 2006).

A resistência insulínica é considerada um fenômeno fisiopatológico no qual há a alteração na ação biológica da insulina e nos diferentes tecidos periféricos levando a uma hiperinsulinemia compensatória. Quando o organismo não pode mais manter a resposta a essa hiperinsulinemia há o desenvolvimento da Diabetes Mellitus tipo 2. A resistência insulínica representa uma evidência na maioria das pessoas portadoras de síndrome metabólica (PINEDA, 2008).

Segundo Castro et al. (2014), outros órgãos como: vasos (resultando em vasoconstricção/hipertensão), cérebro (levando à maior ingestão calórica), pâncreas (diminuição de massa e sensibilidade à glicose das células Beta) e ossos (possível diminuição de massa e força), também sofrem alterações devido às consequências resultantes da alteração da ação da insulina.

Alguns autores tais como Carrasco, Galgani e Reyes (2013) definem que a síndrome metabólica é também conhecida como síndrome de resistência insulínica e tal enfermidade é caracterizada por obesidade central, hiperglicemia e dislipidemia, bem como hipertensão arterial, inflamação crônica, além da resistência insulínica propriamente dita, e que todos esses fatores juntos levam a uma maior propensão à doença trombótica.

A resistência insulínica vem sendo estudada desde 1939 pelo autor inglês $\mathrm{H}$. Himsworth, que demonstrou que a absorção de glicose poderia ser variável de um indivíduo para o outro conforme sua sensibilidade celular à insulina. Somente em 1979 De Fronzo, nos Estados Unidos fez um relato circunstanciado de uma técnica específica para medir a resistência à insulina, a qual nomeou de Teste de Fixação 
Euglicêmica da Insulina. Em 1998, a Organização Mundial da Saúde (OMS) criou um critério para a delimitação desse quadro, o qual foi chamado pela primeira vez de síndrome metabólica. O National Institute of Health, via National Colesterol Education Program (NCEP), juntamente com o $3^{\circ}$ Adult Treatment Panel (ATPIII), em 2001, sugeriu um novo critério mais simples, no qual requeria pelo menos três componentes anormais para que o diagnóstico pudesse ser feito. No Brasil, em 2005, foi publicada a I Diretriz Brasileira de Diagnóstico e Tratamento da Síndrome Metabólica com o apoio da Sociedade Brasileira de Cardiologia (LUNA, 2007).

Apesar de ainda existirem debates a respeito da definição de Síndrome Metabólica, seu valor como marcador de risco independente para doenças cardiovasculares (DCV) ainda é alto. Com isso, acredita-se que, políticas públicas realizadas para a prevenção de DCV podem ser otimizadas quando forem voltadas à prevenção e ao tratamento da síndrome metabólica como um todo e não a fatores de riscos individuais (DUTRA, 2011). No Brasil, pode-se observar que está ocorrendo uma transição nutricional, onde o número de pessoas com sobrepeso e obesidade vem aumentando (FERREIRA et al. 2007).

Neste contexto, é inquestionável a importância do desenvolvimento de um estudo sobre a prevalência da resistência insulínica associada ao perfil lipídico e síndrome metabólica, a fim de determinar um perfil dessa enfermidade no Distrito Federal, podendo colaborar com pesquisas de áreas afins e contribuir com possíveis intervenções no monitoramento da resistência insulínica.

\section{OBJETIVOS}

\subsection{Objetivo geral}

Analisar a prevalência dos casos de resistência insulínica $(\mathrm{RI})$ e sua relação com o perfil lipídico no desenvolvimento da Síndrome Metabólica (SM) na população do Distrito Federal, entre os anos de 2015 e 2016, a fim de fornecer subsídios para possíveis intervenções clínicas visando esclarecer, prevenir e orientar a população para os fatores de risco e impacto das doenças cardiovasculares. 


\subsection{Objetivos específicos}

- Analisar a prevalência da resistência insulínica de acordo com a faixa etária e gênero;

- Avaliar o impacto da resistência insulínica pelo índice HOMA - IR, pelos índices de sensibilidade insulínica derivados do TOTG, verificação da hemoglobina glicada como precursores de doença coronariana, diabetes mellitus 2 e obesidade.

- Verificar o perfil lipídico dos pacientes a partir dos resultados obtidos na análise dos níveis de triglicerídeos (TGs), colesterol total e de suas frações;

- Apresentar a prevalência da síndrome metabólica.

\section{FUNDAMENTAÇÃO TEÓRICA}

Os padrões de dietas e exercícios físicos vêm mudando drasticamente ao longo das últimas décadas, com isso, houve um aumento da prevalência da obesidade e suas complicações, sendo a resistência insulínica (RI) a principal delas. O estado de RI é caracterizado pela perda da função total do hormônio que tem como principal função, manter a homeostasia glicêmica, suprimindo a liberação de ácidos graxos, favorecendo a síntese de triglicerídeos no tecido adiposo. Além disso, a insulina inibe a produção de glicose no fígado, por facilitar a captação da mesma no músculo esquelético e no tecido adiposo (CARRASCO; GALGANI; REYES, 2013).

Notavelmente, como afirmado por Castro et al. (2014), o efeito mitogênico da insulina é preservado nos casos de RI, podendo levar a uma predisposição ao câncer. A insulina pode ainda acometer receptores como o IGF-1 de cartilagem, levando a traços de acromegalia, ou nas células da teca do ovário, aumentando a produção de andrógenos podendo causar síndrome do ovário policístico.

Recentemente houve um aumento no número de pesquisas a respeito do papel da RI no organismo. Foi evidenciado sua relação com processo patológico da diabetes, onde a resistência insulínica pode anteceder o quadro de diabetes por $10 \mathrm{a}$ 20 anos. Sendo assim, a avaliação quantitativa da RI pode ser bastante útil para detectar sua presença e gravidade, ainda mais em pacientes que ainda não desenvolveram tolerância à glicose detectável ou diabetes (BORAl et al., 2011). A resistência insulínica, como evento preciso no desenvolvimento da diabetes tipo 2 , 
pode ser provocada pelo acúmulo de lipídeos, que ocasionará a intolerância a glicose e hiperglicemia de jejum (MOSSMANN, 2015).

Segundo Matos (2016), o mecanismo do surgimento da resistência insulínica relacionada à obesidade e perfil lipídico é multifatorial, e ainda não é totalmente elucidada. Uma das hipóteses pode estar relacionada à expansão do tecido adiposo e a infiltração de células inflamatórias exacerbada neste tecido. Isto pode levar à maior liberação de ácidos graxos livres e de citocinas com ação pró-inflamatória como o fator de necrose tumoral alfa (TNF- $\alpha$ ) e a interleucina 1 beta (IL-1ß), estes então atuam em vias de sinalização no músculo esquelético, através de receptores específicos.

Jung \& Choi (2014), reafirmam a proposição anterior esclarecendo que o tecido adiposo, além de ser a principal forma de armazenamento energético do organismo, ele vem sido reconhecido como um órgão endócrino. O tecido adiposo, quando em excesso, pode resultar em uma disfunção de fatores secretados por ele (adipocinas), que contribuem para o surgimento de distúrbios metabólicos, através de alterações na homeostase da glicose e dos lipídeos, além de respostas inflamatórias. Adicionalmente, o acúmulo de gordura em excesso resulta na quebra dos adipócitos e, consequentemente na liberação de ácidos graxos livres (AGL) na circulação. $O$ aumento sérico de $A G L$ pode ser um fator crítico na modulação da sensibilidade à insulina de um tecido.

De acordo com Nelson \& Cox (2014) o estágio intermediário que precede o diabetes tipo 2 pode ser também conhecido como Síndrome Metabólica ou Síndrome X. A síndrome metabólica é caracterizada por um conjunto de fatores de risco cardiovascular relacionados ao acúmulo central de gordura e à RI. A presença de pelo menos três das seguintes alterações classifica o sujeito como portador da síndrome metabólica: obesidade abdominal, elevação de níveis de triglicerídeos, baixas taxas de colesterol de alta densidade (HDL-c), e aumento de pressão arterial e glicemia em jejum. Estes critérios foram definidos pela National Cholesterol Education Program Adult (NCEP-ATP III) (PIMENTA; GAZZINELLI; VELÁZQUEZ-MELÉNDEZ, 2011), a fim de padronizar o diagnóstico da síndrome metabólica internacionalmente, pelo menos para adultos, pois para adolescentes e crianças, os parâmetros ainda não estão bem definidos (CARVALHO et al., 2016). 
O tratamento para a Síndrome Metabólica segundo Hilal-Dandan \& Brunton (2015) deve se concentrar principalmente na perda de peso e no aumento da atividade física assim como no tratamento para anormalidades lipídicas. A restrição alimentar irá reduzir a carga total de ácidos graxos controláveis, e o exercício ativar a AMPK (Activated Protein Kinase) que altera o metabolismo no sentido da oxidação da gordura e ao mesmo tempo inibe a sua síntese.

\section{METODOLOGIA}

Realizou-se uma pesquisa básica quantitativa por meio de um estudo transversal observacional descritivo dos exames realizados pelos pacientes atendidos no laboratório-escola de análises clínicas do UniCEUB entre os anos de 2015 a 2016.

As informações foram coletadas da base de dados eletrônica (Hygia) utilizada pelo laboratório-escola, preservando a identidade do paciente. Os objetos de estudo foram variáveis pré-selecionadas como: sexo, faixa etária, antecedentes epidemiológicos e resultado de exames laboratoriais tais como glicemia em jejum, hemoglobina glicada e TOTG, perfil lipídico, e outras informações que demonstrasse ser importantes para o estudo da resistência insulínica.

Quanto aos valores de referência dos exames, foram utilizados aqueles empregados no laboratório-escola de análises clínicas (tabela 1).

Para a utilização das informações nesta pesquisa, foram selecionados dados de pacientes que apresentavam os seguintes critérios de inclusão:

- Ser paciente atendido no laboratório-escola de análises clínicas do UniCEUB;

- Apresentar idade igual ou superior a 18 anos;

- Ter realizado os exames de glicemia em jejum, hemoglobina glicada, teste oral de tolerância a glicose (TOTG) e perfil lipídico entre os anos de 2015 a 2016.

Quanto aos critérios de exclusão, foram desconsiderados dados de pacientes que apresentavam:

- Idade inferior a 18 anos; 
- Que não tenha realizado todos os exames importantes para esta pesquisa

Por se tratar de um levantamento de dados, os riscos referentes ao voluntário são mínimos. Desta forma, esta pesquisa foi encaminhada para apreciação do comitê de ética em pesquisa do UniCEUB, solicitando a liberação do Termo de Consentimento Livre e Esclarecido (TCLE), uma vez que não havia intensão e nem necessidade de identificação dos pacientes. $O$ trabalho foi aprovado pelo comitê de ética, portando parecer número 1.942.659. 
Tabela 1. Valores de Referência de acordo com o Laboratório Escola do UniCEUB.

\begin{tabular}{|c|c|}
\hline Exame & Valor de referência \\
\hline Glicose & de 70 a $99 \mathrm{mg} / \mathrm{dL}$ \\
\hline $\begin{array}{l}\text { Teste oral de tolerância a } \\
\text { glicose (TOTG 120') }\end{array}$ & $\begin{array}{l}\text { Normal - até } 139 \mathrm{mg} / \mathrm{dL} \\
\text { Alto (Resistente à Insulina) - } 140 \text { a } 200 \mathrm{mg} / \mathrm{dL} \\
\text { Diabetes Melitus - superior a } 200 \mathrm{mg} / \mathrm{dL}\end{array}$ \\
\hline Hemoglobina glicada (HbA1C) & 3,9 a $6,1 \%$ \\
\hline Colesterol total & $<190 \mathrm{mg} / \mathrm{dL}$ \\
\hline HDL- Colesterol & $>40 \mathrm{mg} / \mathrm{dL}$ \\
\hline LDL - Colesterol & $\begin{array}{l}<130 \mathrm{mg} / \mathrm{dL} \text { - Baixo risco cardiovascular; } \\
<100 \mathrm{mg} / \mathrm{dL} \quad-\text { Risco cardiovascular } \\
\text { intermediário; } \\
<70 \mathrm{mg} / \mathrm{dL}-\text { Alto risco cardiovascular; } \\
<50 \mathrm{mg} / \mathrm{dL} \text { - Risco cardiovascular muito alto }\end{array}$ \\
\hline VLDL - Colesterol & $\begin{array}{l}<160 \mathrm{mg} / \mathrm{dL} \text { - Baixo risco cardiovascular; } \\
<130 \mathrm{mg} / \mathrm{dL} \quad-\text { Risco cardiovascular } \\
\text { intermediário; } \\
<100 \mathrm{mg} / \mathrm{dL}-\text { Alto risco cardiovascular; } \\
<80 \mathrm{mg} / \mathrm{dL} \text { - Risco cardiovascular muito alto }\end{array}$ \\
\hline Triglicerídeos & $<150 \mathrm{mg} / \mathrm{dL}$ \\
\hline Insulina & 2,6 a $24,9 \mu \mathrm{U} / \mathrm{mL}$ \\
\hline HOMA-IR & Resistência insulínica: HOMA-IR > 4,65 \\
\hline
\end{tabular}




\subsection{Coleta e geração de dados}

Os dados selecionados foram registrados em uma planilha visando identificar, determinar, caracterizar e conhecer as temáticas tratadas através de um quadro esquemático.

Para o cálculo da prevalência foi utilizada a seguinte fórmula:

$$
\frac{\mathrm{N}^{\circ} \text { de indivíduos afetados }}{\text { POPULAÇÃO TOTAL }}
$$

As análises foram realizadas empregando-se planilhas do Excel, seguida pela produção de elementos gráficos que demonstram de forma fidedigna, o que foi observado.

\section{FUNDAMENTAÇÃO TEÓRICA}

Foram analisados 217 documentos, referentes a 64 homens (29\%) e 153 mulheres $(71 \%)$ com idade compreendida entre 19 a 90 anos.

A resistência insulínica $(\mathrm{RI})$ foi determinada com base nos resultados dos exames de teste oral de tolerância a glicose (TOTG), uma vez que este exame estava presente em todos os documentos selecionados. O índice HOMA-IR, considerado o exame mais indicado para o diagnóstico da RI segundo Borai (2011) e Carvalho (2016), esteve presente em apenas 33 documentos o que inviabiliza seu uso como parâmetro diagnóstico único, nesta pesquisa.

A prevalência da $\mathrm{RI}$ foi de $30,41 \%$. Ao analisarmos a prevalência entre os sexos e intervalos de idade, observamos que: a prevalência para RI foi de $23,44 \%$ para homens e 33,3\% para mulheres; e de 9,1\% para indivíduos com idade entre 19 a 35 anos e de $33,15 \%$ para as idades compreendidas entre 35 e 90 (figura 1). 


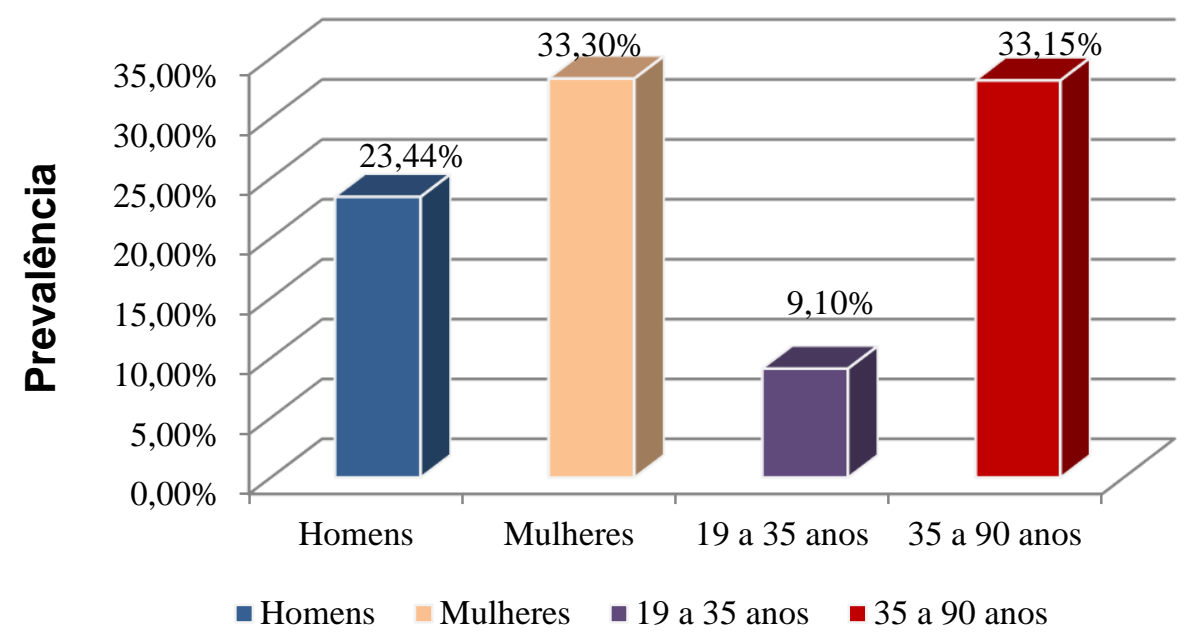

Figura 1: Prevalência da resistência insulínica em homens, mulheres e entre as faixas etárias de 19 a 35 anos e 35 a 90 anos.

Em estudos realizados em populações brasileiras a prevalência da RI foi de 13,1\% em uma população com 151 sujeitos (CARDOSO; MATTOS; KOIFMAN, 2005). Já em um estudo realizado no Espírito Santo, a prevalência de RI foi de 10,4\% em 501 sujeitos avaliados (SALAROLI, 2011). Estes resultados foram significativamente menores do que o valor encontrado neste estudo, o que pode ser explicado pela diferença de parâmetros de diagnóstico utilizados e no tamanho da amostra.

O aumento da prevalência em mulheres pode ser explicado pelo fato deste grupo compor $71 \%$ da amostra. Porém, a prevalência da RI em mulheres quando comparadas ao sexo masculino também foi elevada em estudo chinês, que avaliou 2142 indivíduos (CHEN et al., 2013) e no estudo brasileiro, que avaliou 151 indivíduos (CARDOSO, MATTOS \& KOIFMAN, 2005). Este fato pode ser explicado pelos fatores hormonais femininos e o hipometabolismo (OTT et al., 2011).

Já o fato de a prevalência da RI em indivíduos da faixa etária de 35-90 ser maior do que em indivíduos mais jovens pode ser justificado, com a teoria de que com o avançar da idade, indivíduos tendem a acumular mais fatores que favorecem a manifestação da síndrome da resistência à insulina (CARDOSO, MATTOS \& KOIFMAN, 2005).

Quanto ao perfil lipídico foi possível observar que dos documentos analisados $68(31 \%)$ apresentaram alteração nos níveis de triglicerídeos (TG) (maior que 150 
$\mathrm{mg} / \mathrm{dL}$ ), 124 (57\%) nos níveis de colesterol total (maior ou igual a $190 \mathrm{mg} / \mathrm{dL}$ ), $53 \mathrm{com}$ níveis de HDL abaixo de $40 \mathrm{mg} / \mathrm{dL}$; 83 com níveis de porção não-HDL elevados (maior que $130 \mathrm{mg} / \mathrm{dL}$ ). Do total de documentos, 21 (9\%) pacientes apresentaram alteração concomitante em todos os parâmetros analisados (figura 2).

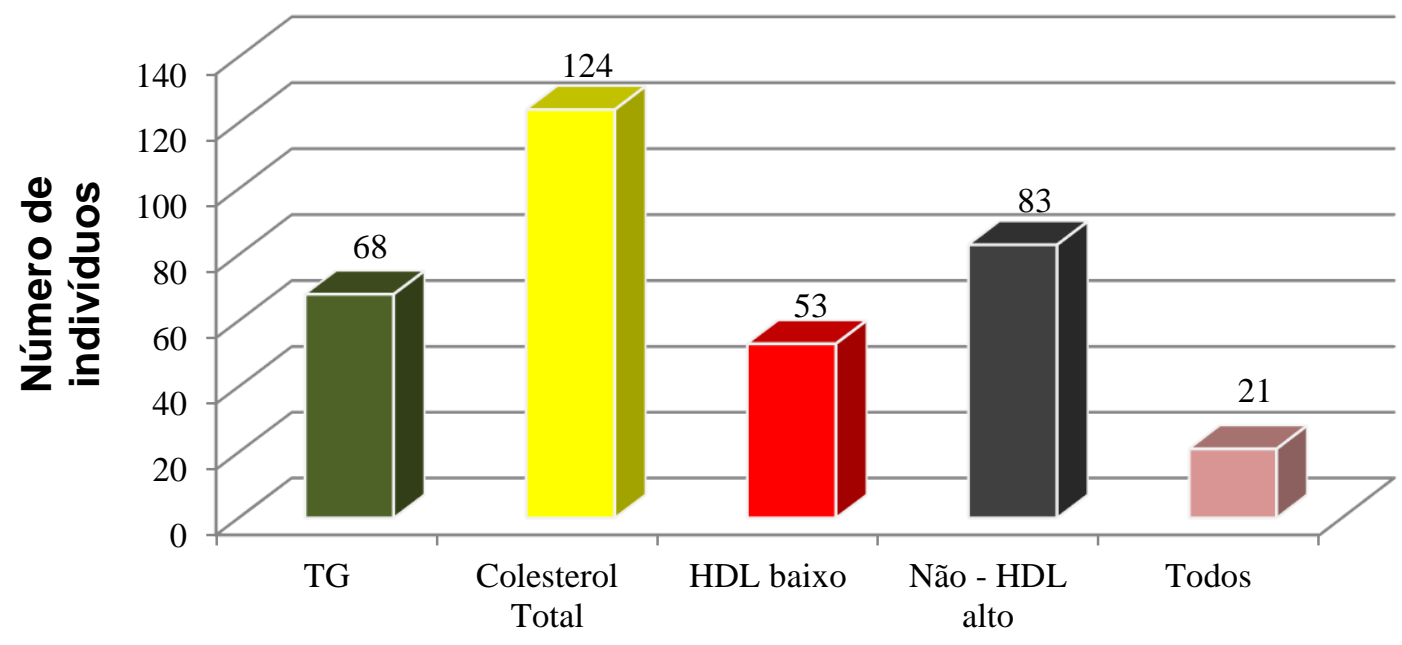

Figura 2: Perfil lipídico da população estudada. TG. Triglicerídeos $>150 \mathrm{mg} / \mathrm{dL}$; colesterol total ( $\geq 190 \mathrm{mg} / \mathrm{dL}$ ), colesterol $\mathrm{HDL}<40 \mathrm{mg} / \mathrm{dL}$, porção não $\mathrm{HDL}$ (> $130 \mathrm{mg} / \mathrm{dL}$ )

O perfil lipídico é formado pela análise dos exames de triglicerídeos e colesterol total, que são os principais lipídeos encontrados no plasma humano. Estes são transportados por lipoproteínas, sendo as principais HDL, LDL e VLDL. Os triglicerídeos e o colesterol entram no plasma na forma de pequenas partículas lipoproteicas ricas em triglicerídeos (VLDL e quilomícron), sendo então hidrolisadas por enzimas intravasculares em ácidos graxos e monoglicerídeos. O colesterol é o principal componente da LDL, sendo então um menor componente do VLDL e HDL. O HDL faz o transporte reverso do colesterol, removendo o excesso de colesterol dos tecidos extra-hepáticos e levando-os de volta para o fígado para que possam ser eliminados através da bile. Sendo essa função imprescindível para a inibição da aterogênese (SCHIAVO, LUNADERLLI \& OLIVEIRA, 2003).

A alteração dos níveis de perfil lipídico da amostra estudada é preocupante, pois o aumento de triglicérides está associado aos riscos de doenças coronarianas, 
por contribuir à aterogênese da maneira direta e influenciarem o tamanho e concentração das moléculas de VLDL. Os altos níveis de LDL e baixas taxas de HDL estão relacionadas à manifestação de doenças cardíacas. O ideal é manter os níveis de colesterol total baixos e também de LDL mas, manter a concentração de HDL 0 mais alta possível (SCHIAVO; LUNADERLLI; OLIVEIRA, 2003).

Ao associarmos os dois parâmetros ( $R \mid$ e alteração em algum dos marcadores de perfil lipídico) foi possível observar que 58 pacientes (prevalência de 27\%) tinham alteração concomitante. Esse fato nos leva a imaginar que os indivíduos estão predispostos a apresentar doenças coronarianas, diabetes mellitus tipo 2 e obesidade, caso já não manifestem.

A associação entre resistência insulínica e o perfil lipídico pode ser explicada pelo acúmulo de gordura abdominal (obesidade central). Segundo Lerario et al., (2002), os triglicerídeos distribuídos no abdômen possuem turnover (capacidade de quebra/ transformação) mais rápido, aumentando assim a oferta de ácidos graxos livres no organismo, estimulando a gliconeogênese e inibindo a filtração de insulina pelo fígado. Com isso, os TGLs contribuem para a elevação da glicemia, insulinemia e consequentemente a RI. Embora não tenhamos os dados do percentual de gordura abdominal e nem o perfil corporal dos pacientes analisados, vale ressaltar que esta gordura está associada ao risco cardiovascular e a distúrbios metabólicos.

Bonora et al. (1998) identificaram maiores ocorrências de RI nos indivíduos com hipertrigliceridemia ou naqueles com baixos níveis de HDL-C do que em indivíduos com o colesterol elevado, sugerindo então que a hipercolesterolemia está menos associada à RI do que as outras alterações. Oliveira et al. (2007) identificaram níveis de HOMA-IR mais alterados nos indivíduos com hipertrigliceridemia em concordância com Bonora et al., embora esta concordância não se aplique ao HDLC. Já Vasques et al. (2009), encontraram mais correlação entre a RI e o HDL-C. Além destes, estudos como os de McLaughlin et al. (2005), Hannon et al. (2006), Sumner \& Cowie (2008) também estabelecem a relação entre marcadores de perfil lipídicos alterados com a presença de resistência à insulina.

Segundo a literatura, um indivíduo que apresente alteração no metabolismo de carboidrato (glicemia) associado à alteração no perfil lipídico (triglicerídeos e HDL-c) já é considerado como um portador de síndrome metabólica (SM). De acordo com os 
critérios utilizados pelo National Cholesterol Education Program - Adult Treatment Panel III (NCEP-ATPIII), para a manifestação da SM o paciente precisa apresentar alteração em pelo menos três parâmetros dos relacionados a seguir: circunferência abdominal maior que $102 \mathrm{~cm}$ para homens e $88 \mathrm{~cm}$ para mulheres, pressão arterial maior ou igual a $130 / 85 \mathrm{mmHg}$, glicemia maior ou igual a $100 \mathrm{~mm} / \mathrm{dL}$, triglicerídeos maiores ou iguais a $150 \mathrm{mg} / \mathrm{dL}$ e colesterol HDL menor que $40 \mathrm{mg} / \mathrm{dL}$ para homens e $50 \mathrm{mg} / \mathrm{dL}$ para mulheres (PIMENTA; GAZZINELLI; VELÁZQUEZ-MELÉNDEZ, 2011).

Com base nos dados coletados observamos que 28 indivíduos apresentam critérios favoráveis à manifestação da SM (prevalência de 13\%). Para este resultado foram utilizados os dados de glicemia, triglicerídeos e HDL.

Como já mencionado, diversos outros parâmetros são utilizados para o diagnóstico da SM, entretanto, estes dados não constavam nos documentos analisados, o que pode comprometer a análise, uma vez que, a circunferência abdominal, por exemplo, é considerada um dos indicadores com o maior valor preditivo para a síndrome metabólica. Porém, como já foi estabelecido, contanto que o paciente se enquadre em quaisquer 3 parâmetros dos 5 estabelecidos pelo NCEPATPIII, ele é considerado um portador da síndrome (SALAROLI et al., 2007).

De acordo com Dutra (2011) na região central do Brasil, a prevalência da síndrome metabólica, em diferentes populações estudadas pode variar de 19 a $25 \%$. Estes valores não fogem muito do encontrado no atual estudo. No entanto, no estudo realizado pelo autor, a prevalência foi de $32 \%$ na população do Distrito Federal. Em sua discussão, o resultado obtido por Dutra (2011) é concordante com os resultados dos Estados Unidos (34\%). Em países com situação econômica similar à nossa, os valores de Dutra (2011) são muito próximos dos da Venezuela $(31,2 \%)$, maiores que os do Peru $(26,9 \%)$ e menores que a prevalência encontrada na Índia $(41,4 \%)$. A diferença dos valores pode ser justificada pelo número de sujeitos do estudo (2170) ter sido maior do que a amostra utilizada no atual estudo $(n=217)$. Outro fator que pode justificar a pequena discrepância é o fato de que mais parâmetros foram utilizados para o estabelecimento do diagnóstico da síndrome, como a circunferência abdominal, pressão sistólica e diastólica. A prevalência de 13\% encontrada no estudo realizado continua sendo um valor alarmante que comprova a crescente de distúrbios metabólicos. 
A forma de tratamento da SM é baseada na perda de peso, sendo a perda de 7 a $10 \%$ do peso inicial suficiente para se obter uma melhora na circunferência abdominal, perfil lipídico e glicemia (LOTTEMBERG, GLEZER \& TURATTI, 2007). Para Ciolac \& Guimarães (2004), alguns estudos epidemiológicos demonstram forte relação entre a falta de atividade física e a presença de fatores de risco para a síndrome metabólica. Assim, a prática de atividades físicas regulares tem uma excelente contribuição para a prevenção e tratamento desta síndrome.

O aumento da prevalência de Síndrome Metabólica, torna cada vez mais evidente a necessidade de incentivo para o desenvolvimento de novas políticas públicas que favoreçam a diminuição de casos. Afinal, já é estabelecido que a SM é um fator de risco para o desenvolvimento de doenças cardiovasculares e outras complicações, necessitando de novos protocolos e procedimentos de prevenção dos fatores de risco como um todo, de modo que eles parem de ser abordados separadamente (DUTRA, 2011).

Embora os achados deste trabalho sejam relevantes e apresentem contribuição científica, a falta de dados na dosagem de insulina em muitos prontuários pode ter impactado na prevalência da síndrome bem como na dificuldade de comparação com outros estudos que utilizam o cálculo do HOMA-IR como principal e melhor diagnóstico para esta patologia. Assim, recomenda-se estudos futuros com nova análise dos prontuários do Laboratório Escola do UniCEUB, com pacientes atendidos no último ano e que tenham feito os exames de insulina e/ou HOMA-IR, bem como a comparação entre os métodos diagnósticos TOTG e HOMA-IR quanto à eficácia na identificação da $R I$ e avaliação da sua prevalência utilizando os dois tipos de diagnóstico.

\section{CONSIDERAÇÕES FINAIS}

A prevalência da $\mathrm{RI}$ foi alta, especialmente quando comparada a outros estudos. Foi possível perceber também que mulheres são mais afetadas quando 
comparadas a homens, fato este visto em outros ensaios clínicos. Já pessoas com idade avançada tendem a manifestar mais a RI, devido ao acúmulo de fatores ao longo da vida, que culminam no desenvolvimento da mesma. Ao associar RI e algum marcador do perfil lipídico alterado dos pacientes selecionados, o valor da prevalência é alto, o que indica que estes indivíduos têm maior risco a manifestar alterações cardiovasculares, peso elevado e doenças graves como diabetes mellitus tipo 2.

Embora o HOMA-IR seja considerado o melhor método diagnóstico, o número reduzido de pacientes que realizaram o HOMA-IR não impossibilitou a análise da prevalência da RI, feita por meio do exame de TOTG. Com base neste exame é possível avaliar uma predisposição para o desenvolvimento da RI no paciente e tomar precauções para evitar este evento.

A alta prevalência de alterações no perfil lipídico indica que os indivíduos analisados possuem alto risco de progredir para a RI, caso já não manifeste, devido a forte ligação em alterações de marcadores lipídicos e a presença de RI. Não foi possível ter conhecimento se os pacientes selecionados que possuem alguma alteração no perfil lipídico, já manifestam alguma patologia ou alteração coronariana.

A SM não foi tão prevalente nesta população quanto a RI, mas a relevância de sua presença é inquestionável e requer atenção para que estes valores não fujam do controle. Medidas de precaução devem ser tomadas para controle no aumento do número de casos destes distúrbios. A população do Distrito Federal, apesar de não manifestar prevalências alarmantes da RI e SM, precisa de atenção e cuidados para o manejo e controle dessas síndromes, buscando conscientizar a população a respeito da importância de uma vida saudável.

O governo e veículos midiáticos podem utilizar as informações obtidas através de estudos científicos como principal motivador para incentivar a população a cuidar da alimentação e utilizar de atividades físicas como aliados para a garantia de um organismo saudável, evitando ou protelando a manifestação da RI e SM, e consequentemente das temidas doenças cardiovasculares/coronarianas.

\section{REFERÊNCIAS}

BORAI, A. et al. Selection of the appropriate method for the assessment of insulin resistance. BMC medical research methodology, v.11, n.1, p.158, 2011. 
CAMPOS, K. E. et al. Obesidade e resistência à insulina. Femina. Vol 34, № 9, 2006. Disponível em: <http://www.febrasgo.org.br/site/wpcontent/uploads/2013/05/Femina_34-9-15.pdf> Acesso em 24 abr 2016.

CARDOSO, A. M.; MATTOS, I. E.; KOIFMAN, R. J. Prevalência de diabetes mellitus e da síndrome de resistência insulínica nos índios Guaraní do Estado do Rio de Janeiro. Coimbra Júnior. CEA, Santos RV, Escobar AL, organizadores. Epidemiologia e Saúde dos Povos Indígenas no Brasil. Rio de Janeiro: Editora Fiocruz, p.169-185, 2003.

CARRASCO, F; GALGANI, J. E.; REYES, M. Síndrome de resistencia a la insulina. Estudio y manejo. Rev. Med. Clin. Condes, 2013. Disponível em: $<\mathrm{http}: / /$ apps.elsevier.es/watermark/ctl_servlet? $\mathrm{f}=10$ \&pident_articulo=90360787\&pid ent_usuario $=0 \&$ pcontactid $=\&$ pident_revista $=202 \&$ ty $=112 \&$ accion $=$ L\&origen $=$ zonadel ectura\&web=www.elsevier.es\&lan=es\&fichero=202v24n05a90360787pdf001.pdf> Acesso em: 02 mai 2016.

CARVALHO, R. B. N. et al; Fatores de risco associados ao desenvolvimento da síndrome metabólica em crianças e adolescentes. Acta Paulista de Enfermagem, v.29, n.4, 2016.

CASTRO A. V. B. et al. Obesity, insulin resistance and comorbidities-mechanisms of association. Arquivos Brasileiros de Endocrinologia e Metabologia, vol. 58, no. 6, pp. 600-609, 2014

$\mathrm{CHEN}$, S. et al. Insulin resistance and metabolic syndrome in normal-weight individuals. Endocrine, v.46, n.3, p.496-504, 2014.

CIOLAC, E. G.; GUIMARAES, G. V. Exercício físico e síndrome metabólica. Rev Bras Med Esporte, Niterói , v. 10, n. 4, p. 319-324, Aug. 2004 . Available from: <http://www.scielo.br/scielo.php?script=sci_arttext\&pid=S151786922004000400009\&lng=en\&nrm=iso >. access on 20 Ago. 2017.

DUTRA, E. S. Síndrome metabólica no Distrito Federal: prevalência e fatores associados. 2011.

FERREIRA A. P.; OLIVEIRA C. E. R.; FRANCA N. M. Metabolic syndrome and risk factors for cardiovascular disease in obese children: the relationship with insulin resistance (HOMA-IR). J. Pediatr. 2007; 83(1):21- 26.

HANNON, T. S. et al. Use of markers of dyslipidemia to identify overweight youth with insulin resistance. Pediatr Diabetes. 2006; 7:260-6. Disponível em: < https://www.ncbi.nlm.nih.gov/pubmed/17054447 > Acesso em 22 ago 2016. 
HILAL-DANDAN, R.; BRUNTON, L.L. Manual de Farmacologia e terapêutica de Goodman \& Gilman. 2 ed. Porto Alegre: Artmed, 2015.

JUNG, U. J.; CHOI, M. Obesity and its metabolic complications: the role of adipokines and the relationship between obesity, inflammation, insulin resistance, dyslipidemia and nonalcoholic fatty liver disease. International journal of molecular sciences, $v$. 15, n.4, p.6184-6223, 2014.

LERARIO, D. D. G. et al. Excesso de peso e gordura abdominal para Síndrome Metabólica em nilo-brasileiros. Revista Saúde Pública, 2002. Disponível em: <http://www.scielo.br/pdf/rsp/v36n1/8109.pdf> Acesso em: 24 abr 2016.

LOPES, M. J. S. Prevalência da Síndrome Metabólica no Brasil: um estudo de revisão. UEM, 2009. Disponível em: www.def.uem.br/geraMonografia.php?id=72. Acesso em 02 mai 2016.

LUNA, R. L. Síndrome Metabólica. Arquivo Brasileiro de Cardiologia, 2007. Disponível em: $\quad<h t t p: / / w w w . s c i e l o . b r / s c i e l o . p h p ? s c r i p t=s c i=a r t t e x t \& p i d=S 0066$ 782X2007000500027> Acesso em 02 maio 2016.

MATOS, M. A. O Efeito Do Treinamento Intervalado De Alta Intensidade Emcomponentes Celulares E Moleculares Relacionados Á Resistência À Insulina Em Indivíduos Obesos. 2016. Tese de Doutorado do Programa Multicêntrico de PósGraduação em Ciências Fisiológicas da Universidade Federal dos Vales do Jetiquinhonha e Mucuri, Diamantina, 2016.

MCLAUGHLIN T, R. G. et al. Is there a simple way to identify insulin-resistant individuals at increased risk of cardiovascular disease?. Am J Cardiol. 2005; 96:399404. Disponível em: <https://www.ncbi.nlm.nih.gov/pubmed/16054467> Acesso em 22 ago 2016.

MOSSMANN, M. Associação entre resistência insulínica e a doença arterial coronariana em pacientes não diabéticos. Faculdade de Medicina - UFRGS, 2015. Disponível em:

http://www.lume.ufrgs.br/bitstream/handle/10183/129606/000973374.pdf?sequence= 1> Acesso em 03 maio 2016.

NELSON, D.L.; COX, M. M. Princípios de Bioquímica de Lehninger. 6 ed. Porto Alegre: Artmed, 2014.

OTT, J.N. et al.Determinação da Prevalência de Síndrome Metabólica em Mulheres Pós-menopausa da Zona Rural de Catuíbe/RS. Revista Contexto \& Saúde. ljuí, v. 10, n. 20, jan-jun, 2011. 
PIMENTA, A. M.; GAZZINELLI, A.; VELÁSQUeZ-MELÉNDEZ, G. Prevalência da síndrome metabólica e seus fatores associados em área rural de Minas Gerais (MG, Brasil). Ciência \& Saúde Coletiva, v.16, n.7, 2011.

PINEDA, C. A. Síndrome metabólico: definiria, historia, criterios. Colombia Médica. Vol. $39 \quad \mathrm{n}^{\circ} \quad 1, \quad 2008.2$ Disponível em: <http://colombiamedica.univalle.edu.co/index.php/comedica/article/view/556/949> Acesso em 02 de maio 2016.

SALAROLI, L. B. et al. Prevalência de síndrome metabólica em estudo de base populacional, Vitória, ES-Brasil. Arq Bras Endocrinol Metab, p.1143-1152, 2007.

SALAROLI, L. B. Síndrome Metabólica, Resistência à Insulina e Fatores Associados: Um Estudo em Funcionários de uma Rede Bancária da Grande Vitória/Es, Brasil. 2011. Tese de Doutorado do Programa de Pós-Graduação em Ciências Fisiológicas da Universidade Federal do Espírito Santo, Vitória, 2011.

SCHIAVO, M.; LUNARDELLI, A.; OLIVEIRA, J. R. Influência da dieta na concentração sérica de triglicerídeos. J. Bras. Patol. Med. Lab., Rio de Janeiro, v.39, n.4, p.283288, 2003.

SUMMER A.E.; COWIE C.C. Ethnic differences in the ability of triglyceride levels to identify insulin resistance. Atherosclerosis. 2008; 196:696-703. Disponível em: <https://www.ncbi.nlm.nih.gov/pubmed/17254586 > Acesso em 20 ago 2017.

VASQUES, A. C. J. et al. Indicadores do perfil lipídico plasmático relacionados à resistência à insulina. Revista da Associação Médica Brasileira, 2009. 